\title{
A preliminary study of a citizen participation system based on consensus for Decision-making Processes
}

\author{
Francisco Mata \\ University of Jaén, Spain \\ fmata@ujaen.es
}

\author{
Angeles Verdejo \\ University of Jaén, Spain \\ mverdejo@ujaen.es
}

\author{
Luis Pérez \\ University of Jaén, Spain \\ lgonzaga@ujaen.es
}

\author{
Carlos Porcel \\ University of Granada, Spain \\ cporcel@decsai.ugr.es
}

\begin{abstract}
In this work, we present a novel approach to a citizen participation system based on consensus reaching processes in the context of Smart Cities. One of the characteristics of the Smart Cities is to involve the citizens in their community's decision-making. The system that we propose here, allows government representatives to raise proposals to the citizen and permit citizens to give their opinions about the proposed problems. As a novelty, citizens can express their opinions with different preference structures (preference orderings, utility values, and fuzzy linguistic preference relations) to make more flexible the system and to close the opinion expression domains to the knowledge degree of users. Moreover, the decision-making is carried out by means of a consensus reaching process to achieve a minimum level of agreement before making a decision. In this way, the final decisions will be better accepted by citizens. To present this preliminary approach, functional and non-functional system requirements along with a few use cases according to the classical development of Software Engineering are shown.
\end{abstract}

\section{Introduction}

The irruption of new technologies (Internet of Things, Big Data, Mobile Applications, Industry 4.0, ...) and the extension of other existing technologies to other contexts are making possible to improve the efficiency in the management of different types of resources and services in cities, from traffic management, water consumption, etc., to the provision of innovative services such as Smart Government [1]. In the literature we can find numerous references to the characteristics that a city must satisfy to be considered as intelligent [2], highlighting the concept of Smart Governance as one of them, i.e., the design of channels and platforms of participation that allow interaction between citizens, public officials, and decision-making administrations. It is precisely in this scope where we put forward our research work. This new approach to decision-making in smart cities, unlike what is customary, where decisions are made by a very small number of people (politicians, experts, ...), should be participatory and involve both citizens and other agents present in a city (public, private, civil, etc.)

Intelligent Governance can be seen as a step beyond the concept of E-government in which for the automation of administrative processes, new quality public services are incorporated, supported by open technologies and standards that allow the efficient management of resources and services provided to citizens $[3,4,5]$. In addition, citizens must assume an active role in decision-making, contributing proposals that may arise from dialogue, discussion and sharing. All the elements included in this definition must be supported by Information and Communication Technology (ICT) and the Internet of Things (IoT), from the collection of information through electronic devices or platforms, to the extraction of knowledge by applying Big Data technologies to the information collected.

Regarding decision making, this is a complex process and one of the fundamental activities of human beings that we carry out every day consciously or unconsciously [6]. A particular case of decision making is when the process implies more one person, i.e., when several people have to make a decision together. This kind of decision-making processes is known in the literature as group decision making processes. Group decision making (GDM) problems may be defined as decision situations where several people (commonly called decision-makers or experts) attain to reach a common solution to a problem from their opinions or preferences. So, given a set of alternatives, experts try together to find the best alternative to solve the problem.

Another important aspect related to GDM processes is how decision-makers give their opinions, that is, which information domain use to express their preferences about the proposed alternatives. In many cases, it may be advisable that decision-makers express their points of view through domains more consistent 
with either the nature of the alternatives or with their area of expertise. For example, experts of different departments of a company (marketing, accounting, psychology, ...) may prefer to show their opinions using information domains closer to their knowledge fields. Moreover, in decision problems, we can deal with alternatives whose nature is quantitative and others whose nature is qualitative. The first ones can be assessed through precise values but the second ones, related to qualitative aspects, where the uncertainty appears, other types of assessments like linguistic expressions, could be more suitable [7, 8, 9]. Fuzzy Linguistic Approach [10] is a discipline that proposes to use linguistic information (i.e. words) to hand this type of uncertainty and makes decision-making problems more flexible and reliable when people's feelings and opinions are involved.

A simple way to solve a GDM problem is to carry out a selection process where the experts get the best set of alternatives from the preferences expressed by themselves [11]. However, it may happen that some experts consider that their preferences have not been taken into account to obtain the solution and therefore may disagree with that solution. To avoid this situation, a consensus process is advisable (see Fig. 1) where experts discuss and change their preferences to reach enough agreement before making the selection process $[12,13,14]$.

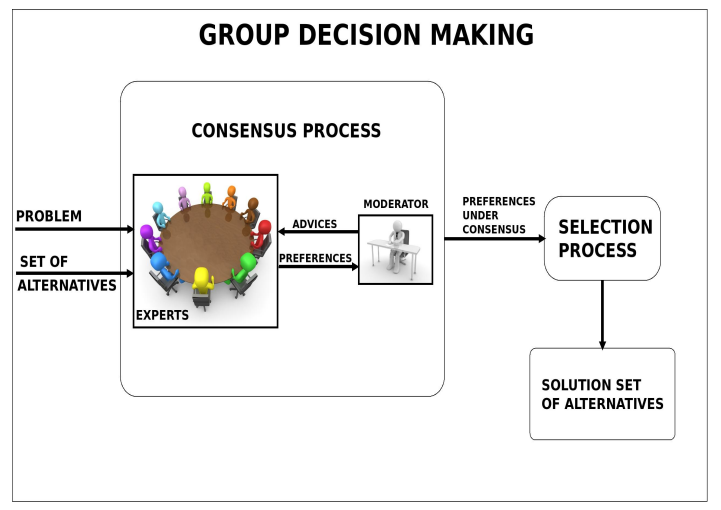

Figure 1. Group decision-making process based on consensus

The consensus is an interesting research area in decision making that has been approached from different points of view $[15,16,17,18,19,20]$. It can be defined as a mutual-agreement state among the members of a group where all opinions have been expressed and listened to the satisfaction of the group [21]. The consensus-building process is a dynamic and iterative process consisting of several rounds, where experts express and discuss their points of view in order to achieve a common solution. Traditionally this process is coordinated by a human moderator, which evaluates the agreement among experts in each round using different consensus measures [22, 23]. If the agreement is not enough, the moderator encourages experts to change their preferences further from the group's opinion in an effort to make them closer in the next consensus round [24].

Therefore, taking into account all these ideas, the aim of this work is to present a novel approach to a citizen participation platform in the Smart City context to carry out decision-making processes based on consensus with fuzzy information. There exist websites where users can evaluate initiative of different nature using the typical "like button" or simple scales of the type Linkert, where the final results are obtained by adding the number of likes or by means of the average of the assessments. As novelties of this work, i) we propose to customize the users' inputs, permitting different preferences structures and information domains, which are handled from the fuzzy logic perspective, in particular fuzzy linguistic approach, and ii) to solve the posed problems by carrying out a consensus reaching process.

The work is structured as follows. Section 2 is dedicated to Preliminaries, where we briefly introduce the theoretical basis that supports our proposal: preference modeling and consensus reaching process performance. In Section 3, the system analysis from the point of view of software engineering is shown, defining user profiles, functional and non-functional requirements and use case diagrams. Finally, Section 4 presents conclusions, future works and open problems.

\section{Preliminaries}

In this section, we introduce the main theoretical concepts supporting our proposal. Firstly, in the subsection called Preference Modeling, the different structures used in the platform to provide the opinions to the system are introduced. Afterward, a brief description of all the steps and phases of the consensus reaching process are shown.

\subsection{Preference Modeling}

In our scope, preference modeling can be defined as the way in which experts express their preferences about the set of alternatives of the problems, i.e., both structures and information domains used by experts to assess their preferences.

In this proposal, to make more flexible the preference modeling, giving experts more freedom to express their opinions, three different structures 
to supply that information are proposed: preference orderings, utility functions and linguistic preference relations. Each one is described following [25]:

- Preference orderings of the alternatives. It is an easy and simple way to represent experts' preferences. The alternatives are ordered from the best to the worst, without any other supplementary information. In this case, an expert, $e_{j}$, provides his preferences on the set of possible alternatives, $X=\left\{x_{1}, \ldots, x_{n}\right\}$, as a preference ordering, $O^{j}=\left\{o^{j}(1), \ldots, o^{j}(n)\right\}$, where $o^{j}($.$) is a permutation function over the$ index set $\{1, \ldots, n\}$ for the expert $e^{j}$ [26]. Hence, according to the point of view of each expert, an ordered vector of alternatives, from the best one to the worst one, is given. So, for every preference ordering $O^{j}$, we will suppose, that the lower the position of an alternative in a preference ordering, implies the better the alternative satisfies the expert and vice versa. For example, let us suppose that an expert $e_{j}$ gives his preferences about a set of three alternatives $X=\left\{x_{1}, x_{2}, x_{3}\right\}$ employing the following ordering preference $O^{j}=\{3,1,2\}$. This means that alternative $x_{3}$ is the best and alternative $x_{2}$ is the worst for that expert.

- Utility functions. In this case, an expert provides a real evaluation (cardinal value) for each alternative, i.e., a function that associates each alternative with a numerical value indicating the assessment of that alternative according to his/her point of view. An utility function can be defined as a set of $n$ utility values $U^{j}=u_{i}^{j}, i=1, \ldots, n, u_{i}^{j} \in[0,1]$, where $u_{i}^{j} \mathrm{k}$ represents the utility evaluation given by the expert $e_{j}$ to the alternative $x_{i} \in X$ [25]. For every set of utility values $U^{j}$, the higher the evaluation is, the better the alternative fulfills the expert's preference.

- Fuzzy linguistic preference relations. In fuzzy contexts, fuzzy preference relations are a popular way to provide experts' preferences [25, 27]. A preference relation may be defined as a matrix $\mathbf{P}_{\mathbf{i}} \subset X \times X$,

$$
\mathbf{P}_{\mathbf{i}}=\left(\begin{array}{ccc}
p_{i}^{11} & \cdots & p_{i}^{1 n} \\
\vdots & \ddots & \vdots \\
p_{i}^{n 1} & \cdots & p_{i}^{n n}
\end{array}\right)
$$

where the value $\mu_{P_{i}}\left(x_{l}, x_{k}\right)=p_{i}^{l k}$ is meant as the preference degree of the alternative $x_{l}$ over $x_{k}$ given by the expert $e_{i}$.

There are many situations in which linguistic assessments are better than numerical assessments. These situations have been addressed from the Fuzzy Linguistic Approach [10], by representing the information as linguistic values by means of linguistic variables. This approach is adequate to qualify phenomena related to human perceptions (e.g., for evaluating the "comfort" of a sofa, terms like "bad", , "tolerable", "good" can be used [28]), or whenever there exists a tolerance for imprecision which can be exploited to achieve robustness and better rapport with reality (e.g., when evaluating the speed of a car, linguistic terms like "fast", "very fast", might be more appropriate that numerical values) [29].

Therefore a fuzzy linguistic preference relation may be defined as:

$$
R: X \times X \rightarrow S
$$

where $S=\left(s_{0}, \ldots, s_{g}\right)$ is the set of linguistic labels whose semantics is defined through fuzzy numbers defined in the $[0,1]$ interval, and $R\left(x_{l}, x_{k}\right)$ is the linguistic preference degree of the alternative $x_{l}$ over $x_{k}$.

\subsection{Consensus reaching process}

In this section, the different phases and steps of the consensus reaching process are briefly introduced. This consensus model has been already proposed to solve problems in other contexts [30]. Now, in this work, we propose as novelty incorporating different ways to provide the decision-makers' preferences according to the three structures introduced in the Preliminaries: preference orderings, utility functions and fuzzy linguistic preference relations. In this way, we achieve major flexibility to express the users' preferences. Experts can choose the way to express their opinions according to their experience or knowledge degree about the problem. It seems logical that experts with a high knowledge degree about a particular problem, are more qualified, for example, for comparing pairs of alternatives by means of preference relations, improving the precision of their preferences. However, experts with less experience can prefer to use more simple representations like preference orderings.

Our model has the following main features:

1. It is able to carry out the consensus process with heterogeneous information (preference orderings, 
utility values, linguistic preferences). To do so, a unification methodology will be required.

2. Two types of measurements are used to evaluate the agreement: proximity measures to measure the distance among experts' opinions and consensus degrees to evaluate the level of agreement among experts.

The consensus process consists of the following phases:

1. Unification of information. In order to operate with the different types of preference modeling, all preferences must be unified into a single domain. We will have to decide if all the numerical preferences are transformed into a linguistic domain or vice versa, by applying techniques of word computing [31, 32].

2. Calculation of the consensus degree. In this phase, the degree of consensus among experts is obtained by computing the similarity among their preferences.

3. Checking the agreement. In this phase, the level of agreement achieved among the experts is checked. Two parameters are required to carry out this task, i) the minimum consensus threshold required before stopping the consensus reaching process, and ii) max-rounds, to avoid an infinite loop and ensuring the end of the process.

4. Generation of recommendations. A set of recommendations with the direction of the changes is suggested with the aim of increasing the agreement in the next round.

A deep description of these phases can be found in the following papers [12, 33, 34].

\section{System analysis}

Once the main purpose of this work has been introduced, we enter the phase of analysis of our system of citizen participation. We will start with a brief introduction to system architecture. Afterward, user profiles that interact with the system are described in detail. We continue presenting the functionality and restrictions of the system and conclude by proposing some use case diagrams according to Unified Modeling Language (UML methodology).

\subsection{System Architecture}

In this section, the main elements of the model are presented along with technologies used to design and to implement the system. As it is depicted in the Fig. 2 , the system has been posed following client-server architecture. So, the system is hosted on a computer which plays the role of internet server ${ }^{1}$. Remote clients can connect with this server through a web browser and HTTP/HTTPS protocols. Users send requests that are parsed on the server and consistent answers are sent back to clients. This architecture frees the end-user of the task of installing the application on his computer. Moreover, it is highly scalable and extensible to add new clients and servers. Note that we propose to employ a database (DB) server to store all the information related to the process, that is, information about problems, experts, preferences, etc, although a unique server might assume both roles, Web and DB server.

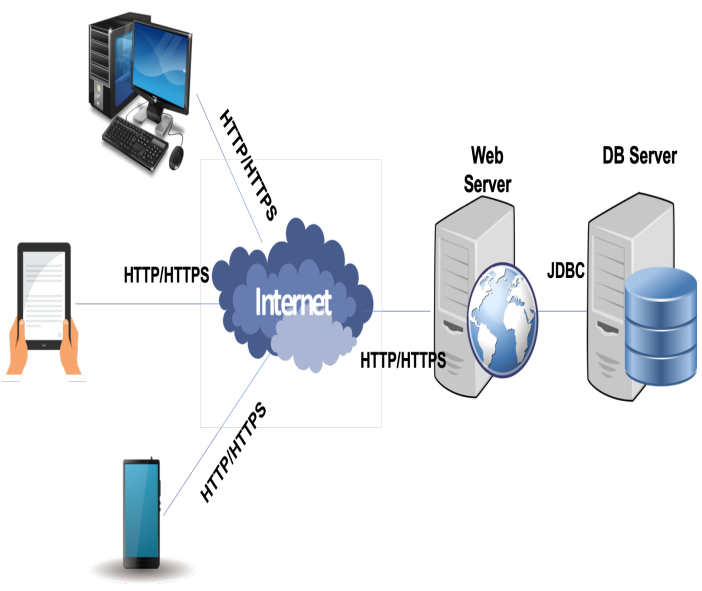

Figure 2. System architecture

\subsection{User profiles}

Before describing both the functional and non-functional requirements of our system, we will define the potential users of our citizen participation platform. As we said before, this is the first approach to a web application prototype, hence new user profiles may appear in the final development. In this analysis state of the system, we have clearly identified two different profiles: Decision-makers, Citizen-users. Next, we will briefly introduce the main characteristics of each profile in order to set functional requirements that will be taken into account in the design of the application and the interface.

1. Decision-makers. This figure represents people that make decisions. In the context of a citizen

\footnotetext{
${ }^{1}$ In this preliminary approach, we are thinking to use Apache web server due to it is open source software, very popular among programmers and used as web server in many organizations.
} 
participation platform, this profile should be assumed by the members of the administration in charge of making proposals for citizen participation. For instance, in the case of a city council, this role would be assumed by the councilors related to citizen participation policies or contact with society. Going deeper into technical aspects, decision-makers need to have a deep knowledge of how the system works. They will be in charge of raising the issues, making decisions regarding the domain of preference expression (numerical, linguistic), fixing the number of alternatives and other aspects related to the consensus and recommendation process (minimum level of agreement, maximum number of rounds, etc.). Moreover, decision-makers should interpret and transmit the solution to the city's government team. From a technical point of view, they should be able to handle computer equipment and knowledge of web application developments would be desirable. Another important aspect of this profile is related to social skills and their ability to interact with their social environment (citizens, NGOs, neighborhood associations, ...), because they must capture the problems and initiatives of citizens and transform them into proposals that can be solved based on the citizen participation platform. They should be able to answer the following two questions:

- What problems are appropriate for the platform?

- How should problems be posed?

2. Citizen-users. This role will be played by the citizens who use the platform. Due to the citizenry is very diverse, there is no specific or concrete profile that characterizes users of the platform, but quite the opposite. The platform might be utilized by citizens of different ages, cultural and educational levels, economic background, ICT skills, and different levels of experience in citizen participation activities. All these aspects have to be taken into account in the platform development. Besides, note the special case of users with some kind of disability who, due to their problems, are usually very participative. This circumstance should be taken into account in order to implement an interface according to their needs and problems.

\subsection{Functional and non-functional requirements}

One of the first steps of a software project according to the principles of Software Engineering is to determine the purpose of the project, the properties that must satisfy and its restrictions.

Requirements of a software project are the set of properties or restrictions that a software project must satisfy. There are two well-differentiated types of such requirements:

a) Functional requirements: those that are specifically related to the operation of the application.

b) Non-functional requirements: those that are related to external factors that may affect or condition the application.

In the following two sub-sections we will define the requirements (both functional and non-functional) of our preliminary proposal. It should be noted that these requirements arise after a detailed analysis of the context of the application and of the functions that we have identified as essential. Probably, when a deeper and more detailed analysis of the system is carried out, new requirements might arise.

The functional requirements describe the functionality of the system, i.e., what the system must provide to users to meet its objectives. Functional software requirements help you to capture the intended behavior of the system. This behavior may be expressed as functions, services or tasks.

3.3.1. Functional requirements. To present them, we have considered suitable to associate them to user profiles that we have introduced previously:

- For all users:

- Identify and validate the user inputs to the system.

- Consult the status of a problem.

- Exit the system.

- For Decision-makers

- Create a problem.

- Modify a problem.

- Consult a problem.

- Delete a problem.

- Create/modify/consult and delete citizen-users. 
- Calculate the level of consensus of the problem.

- Consult the consensus process evolution.

\section{- For Citizen-users}

- Insert opinions or preferences of a problem.

- Consult the status of a problem

- Register in the application

- Modify his/her profile (name, age, marital status, place of residence, etc)

Once the system functions have been defined, we will describe in a more detailed way the response of the system. To distinguish each one of the requirements, we use the identifier, FR- number of requirement:

FR-01: Identify and validate user inputs. The system must be accessible to both user profiles, decision-makers and citizen-users. It must display a form to enter the user name and password. The system will proceed to validate this data by accessing the database that contains information about the different types of users that must have been registered previously. If the information is correct, the user accesses the application, otherwise, it will show an error and will suggest repeating the operation.

FR-02: Exit the system. In the main menu of the application, an option to exit the application will be shown, displaying a form with the possibility to re-enter the system.

FR-03: Create a problem. The system must provide the decision-maker profile the possibility of creating a new problem, specifying the problem identifier, a brief description of the problem objectives, possible alternatives, and any other details deemed appropriate.

FR-04 Consult the status of a problem. The system must allow both decision-makers and citizen-users to consult the description of a problem, the degree of consensus, and recommendations generated by the system in any given round.

FR-05: Modify a problem. The system must allow the modification of a problem stored in the database, add and delete alternatives and modify the parameters of the consensus process (e.g. consensus threshold, number of rounds, etc).

FR-06: Consult a problem. It must permit to consult all the information concerning the problem, such as the identifier, description, alternatives, established parameters, number of citizens participating, etc.

FR-07: Eliminate a problem. It must allow removing a problem in the DB.

FR-08: Modify/consult and delete a citizen-user. The system must allow decision-makers to consult, modify or even delete citizen profiles registered in the DB. This function should not be seen as possible interference in the decision process but as a tool to check the integrity of the information stored in the DB.

FR-09: Assign citizen-users to a problem. It must allow citizens to express their opinions on a list of issues raised. citizen-users should choose the structure that they prefer to give their opinions (utility values, preference orderings or linguistic preference relations).

FR-10: Evaluate and increase the level of agreement of a problem. The system has to unify the information, calculates the consensus degree and generates the necessary recommendations so that the consensus degree is better in the next round.

RF-11: Consult the evolution of the consensus reaching process. The decision-makers should be able to see the evolution of each problem, consult the level of agreement in each consensus round, the citizen-users' preferences and all aspects that provide information and knowledge about the problem.

FR-12: Enter the preferences of a problem. The system must leave the citizen-users to insert their preferences on the problem and display the recommendations of the previous round. It must also allow to accept/reject these recommendations and/or enter new preferences

Non-functional requirements are those that restrict the functional requirements. These requirements normally specify product properties (platform, speed, performance, ...) or restrictions of the graphic interface imposed by the organization (company policies, standards, current legislation, ...).

3.3.2. Non-functional requirements. The non-functional requirements of this proposal have been grouped into requirements of the computer equipment and the application interface. We put 
forward some general requirements although they will have to be defined in more detail as the application development advances.

a) Computer equipment requirements. When we talk about requirements of the computer equipment of a software application developed using Client-Server architecture, we must differentiate between server's requirements and client's requirements.

The clients' equipment requirements, citizen-users in our case, are very simple. They only need a device with Internet access and a web browser. Another aspect to take into consideration is the possibility to access the system from mobile devices (Smart-phones, Tablets, etc.) since this would entail developing an interface according to the responsive web design paradigm.

The requirements of the server, preferably a dedicated web server, are the following. At the hardware level, the computer must be fast enough to run the application in the shortest time possible and with the highest reliability. Many of today's microprocessors are capable of performing this task. Regarding the memory, the equipment must have enough free RAM to perform the operations requested between the application and the database. Concerning the storage, the computer equipment has to storage the DB and allows transactions in a fast and efficient way. We suggest having a dedicated DB server to increase system efficiency. At the software level, it must be able to work on any of the most used operating systems at present. Furthermore., a database management system must be installed, for example, MySQL database. Finally, the server must ensure that the application is accessible via the Internet for all its users 24 hours a day.

b) Interface requirements. The requirements of the graphic interface are closely linked to the usability concept. The usability is defined colloquially as ease of use of a web page, a computer application, or any other system that interacts with a user. From this definition, the basic principles of usability can be obtained, which will be associated with the non-functional requirements that the graphical interface must meet:

- Ease of learning: new users must easily learn to use the application. Important aspects like the generalization of similar tasks with similar executions or the familiarity of the interface have to be taken into account.

- Flexibility: different forms of information exchange between the user and the system. It also covers the multiplicity of ways to accomplish a task.

- Robustness: system's ability to tolerate failures.

\subsection{Use case diagrams}

Use cases can be defined as the representation of users' interactions with the application. A use case is made up of a set of possible sequences of interactions between the system and users in a particular environment and a particular goal. They describe how a task is performed exactly. Therefore, it is necessary to determine which actors are involved in each use case. An actor models an external entity that communicates with the system, i.e., a type of user of the system. An actor, like a use case, must have a unique name and may have an associated description. In our case, we have two actors, Decision-makers, people in charge of raising and evaluating the results of a problem, and Citizen-users, who participate in the consensus process by giving their opinions about the issues raised. The system will have many citizens-users. Once the actors have been defined, we are ready to define the use cases. We have to ensure that all functional requirements defined appear in at least one of the use cases.

In this proposal, we have identified many use cases due to the system functionality is very extensive. For this reason, we have decided to show a few use case diagrams to give an approximate idea of the tasks, activities, and modules of our system. We show the frontier diagram, that completely describes the functionality of the system, and other important diagrams like the problem management, citizen management, consensus process, and problem status.

\section{Conclusions and future works}

A preliminary study of a citizen participation web platform in the Smart Cities context has been presented. This platform could put into practice the concept of Smart Governance, understanding this concept as the involvement of the citizens in the decision-making process of their city.

To make more flexible the way in which citizens can express their opinions or preferences, three different preference structures are proposed: preference 


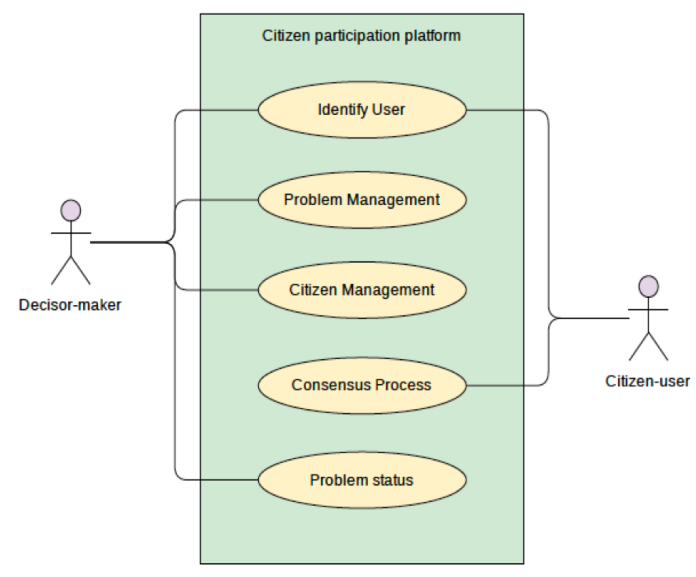

Figure 3. System boundary diagram

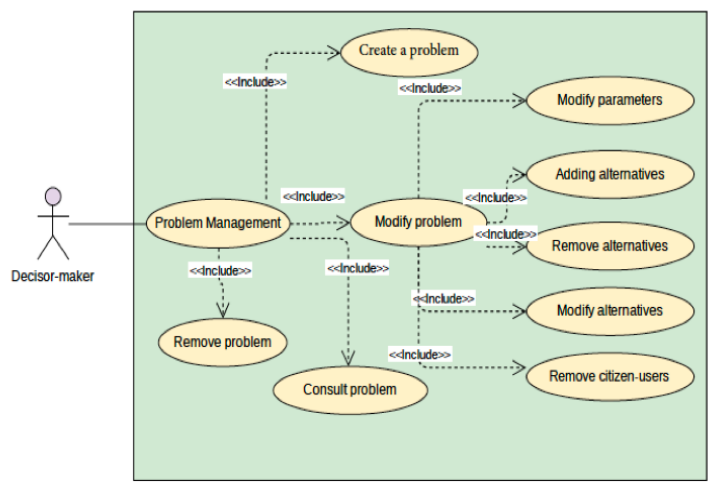

Figure 4. Problem management

orderings, utility values and linguistic preference relations.

Functional and non-functional requirements have been identified and some use case diagrams have been shown by following a classical methodology of software engineering to explain the system functions. Regarding the application interface, some aspects have been introduced from the point of view of application usability. But there are still some open questions that have to be studied in deep, for instance:

- How should the consensus reaching process be? Automatic completely? Semi-supervised?

- How to solve a problem with many citizens? Will the system be efficient? Will some clustering techniques be required?

These open questions can be seen as future works to solve.

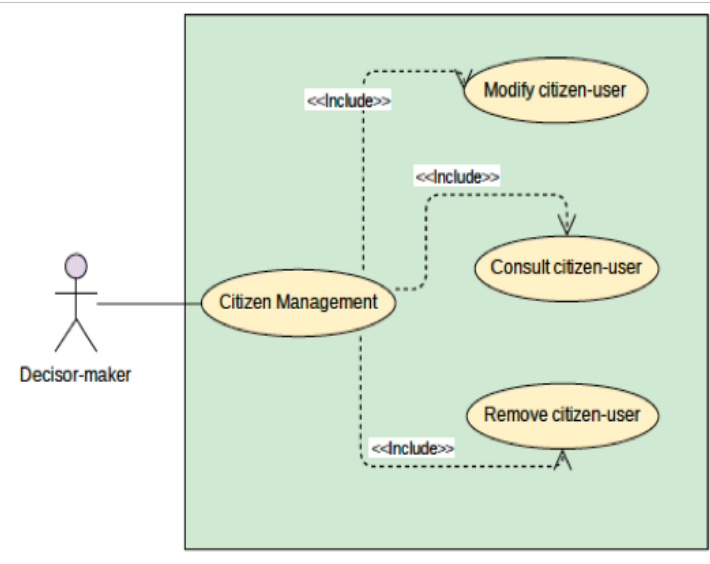

Figure 5. Citizen management

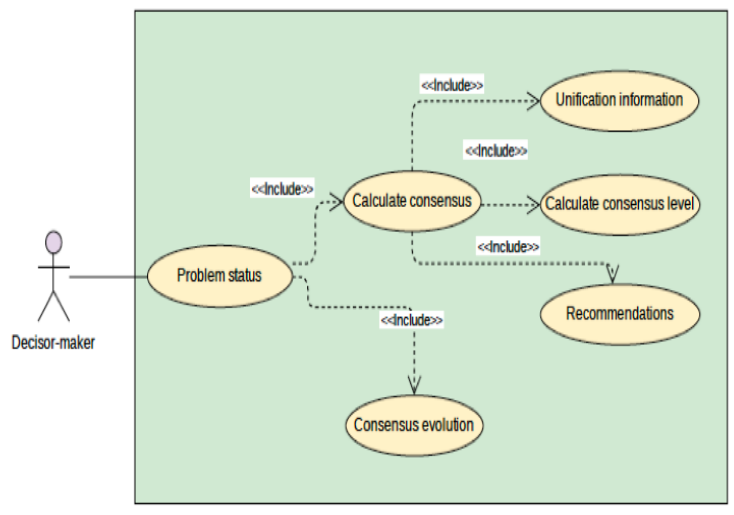

Figure 6. Problem status

\section{Acknowledgment}

This work has been supported by the Spanish State Research Agency through the project PID2019-103880RB-I00 / AEI / $10.13039 / 501100011033$.

\section{References}

[1] S. McClellan, J. Jimenez, and G. Koutitas, Smart Cities. Applications, Technologies, Standards, and Driving Factors. Springer International Publishing, 2018.

[2] H. Scholl and M. Scholl, "Smart governance: A roadmap for research and practice," iConference 2014 Proceedings, 032014.

[3] A. Camero and E. Alba, "Smart City and information technology: A review," CITIES, vol. 93, pp. 84-94, OCT 2019.

[4] M. Rodríguez and L. Alcaide, E-Participation in Smart Cities: Technologies and Models of Governance for Citizen Engagement. Springer International Publishing, 2019.

[5] T. V. Kumar, E-Governance for Smart Cities. Springer Springer Singapore, 2015. 


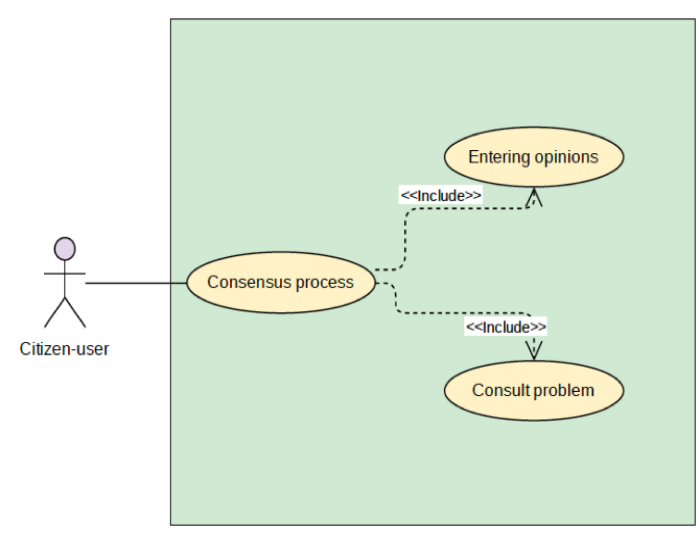

Figure 7. Consensus process

[6] D. Bouyssou and al., Evaluation and Decision Models: A Critical Perspective. Kluwer Academic Publishers, 2000.

[7] F. Herrera and E. Herrera-Viedma, "Linguistic decision analysis: Steps for solving decision problems under linguistic information," Fuzzy Sets and Systems, vol. 115, pp. 67-82, 2000.

[8] R. Yager, "An approach to ordinal decision making," International Journal of Approximate Reasoning, vol. 12, pp. 237-261, 1995.

[9] F. Chiclana, F. Mata, L. G. Pérez, and E. Herrera-Viedma, "Type-1 OWA unbalanced fuzzy linguistic aggregation methodology: Application to Eurobonds credit risk evaluation," International Journal of Intelligent Systems, vol. 33, no. 5, pp. 1071-1088, 2018.

[10] L. Zadeh, "The concept of a linguistic variable and its applications to approximate reasoning," Information Sciences, Part I, II, III, vol. 8,8,9, pp. 199-249,301-357,43-80, 1975.

[11] E. Triantaphyllou, F. Hou, and J. Yanase, "Analysis of the final ranking decisions made by experts after a consensus has been reached in group decision making," Group Decision and Negotiation, vol. 29, no. 2, pp. 271-291, 2020.

[12] E. Herrera-Viedma, L. Martínez, F. Mata, and F. Chiclana, "A consensus support system model for group decision-making problems with multi-granular linguistic preference relations," IEEE Transactions on Fuzzy Systems, vol. 13, no. 5, pp. 644-658, 2005.

[13] E. Herrera-Viedma, F. Herrera, and F. Chiclana, "A consensus model for multiperson decision making with different preference structures," IEEE Transactions on Systems, Man and Cybernetics-Part A: Systems and Humans, vol. 32, no. 3, pp. 394-402, 2002.

[14] J. Kacprzyk, M. Fedrizzi, and H. Nurmi, Consensus Under Fuzziness. Kluwer Academic Publishers, 1997.

[15] F. Cabrerizo, F. Chiclana, I. Pérez, F. Mata, S. Alonso, and E. Herrera-Viedma, "A feedback mechanism based on granular computing to improve consensus in GDM," Studies in Fuzziness and Soft Computing, vol. 357, pp. 371-390, 2018.

[16] A. Kozierkiewicz, M. Pietranik, and M. Sitarczyk, "Updating consensus in case of adding or removing new elements to the profile," Journal of Intelligent \& Fuzzy Systems, vol. 37, no. 6, pp. 7291-7302, 2019.

[17] X. Gou and Z. Xu, Large-Scale Group Consensus Decision-Making Methods with DHHFLPRs, vol. 396 of Studies in Fuzziness and Soft Computing. 2021.

[18] J. García-Lapresta, "Favoring consensus and penalizing disagreement in group decision making," Journal of Advanced Computational Intelligence and Intelligent Informatics, vol. 12, no. 5, pp. 416-421, 2008.

[19] E. Szmidt and J. Kacprzyk, "A consensus reaching process under intuitionistic fuzzy preference relations," International Journal of Intelligent System, vol. 18, no. 7, pp. 837-852, 2003.

[20] Z. Xu, "An automatic approach to reaching consensus in multiple attribute group decision making," Computers and Industrial Engineering, vol. 56, pp. 1369-1374, 2009.

[21] S. Saint and J. R. Lawson, Rules for Reaching Consensus. A Modern Approach to Decision Making. San Francisco: Jossey-Bass, 1994.

[22] E. Herrera-Viedma, F. Mata, L. Martínez, F. Chiclana, and L. Pérez, "Measurements of consensus in multi-granular linguistic group decision making," Lecture Notes in Artificial Intelligence, vol. 3131, pp. 194-204, 2004.

[23] B. Mirkin and T. I. Fenner, "Distance and Consensus for Preference Relations Corresponding to Ordered Partitions," Journal of Classification, vol. 36, no. 2, pp. 350-367, 2019.

[24] S. Zadrozny, An approach to the consensus reaching support in fuzzy environment, pp. 83-109. Kluwer Academic Publishers: Consensus under fuzziness, 1997.

[25] T. Tanino, On Group Decision Making Under Fuzzy Preferences, pp. 172-185. Kluwer Academic Publishers: Multiperson Decision Making Using Fuzzy Sets and Possibility Theory, 1990.

[26] F. Seo and M. Sakawa, "Fuzzy multiattribute utility analysis for collective choice," IEEE Transactions on Systems, Man and Cybernetics, vol. 15, pp. 45-53, 1985.

[27] J. Kacprzyk, "Group decision making with a fuzzy linguistic majority," Fuzzy Sets and Systems, vol. 18, pp. 105-118, 1986.

[28] E. Levrat, A. Voisin, S. Bombardier, and J. Bremont, "Subjective evaluation of car seat comfort with fuzzy set techniques," International Journal of Intelligent Systems, vol. 12, pp. 891-913, 1997.

[29] L. Zadeh, "Fuzzy logic = computing with words," IEEE Transactions on Fuzzy Systems, vol. 4, no. 2, pp. 103-111, 1996.

[30] X. Gou, Z. Xu, and F. Herrera, "Consensus reaching process for large-scale group decision making with double hierarchy hesitant fuzzy linguistic preference relations," Knowledge-Based Systems, vol. 157, pp. 20-33, 2018.

[31] F. Chiclana, F. Herrera, and E. Herrera-Viedma, "Integrating three representation models in fuzzy multipurpose decision making based on fuzzy preference relations," Fuzzy Sets and Systems, vol. 97, pp. 33-48, 1998.

[32] F. Herrera and L. Martínez, "A 2-tuple fuzzy linguistic representation model for computing with words. ieee trans. fuzzy syst. 8(6), 746-752," Fuzzy Systems, IEEE Transactions on, vol. 8, pp. 746-752, 012001. 
[33] F. Mata, L. Martínez, and E. Herrera-Viedma, Intelligent Decision Making: An AI-Based Approach, vol. 97 of Studies in Computational Intelligence, ch. A Consensus Support System for Group Decision Making Problems with Heterogeneous Information, pp. 229-257. Springer, 2008.

[34] F. Mata, L. Martínez, and E. Herrera-Viedma, "An adaptive consensus support system model for group decision-making problems in a multigranular fuzzy linguistic context," IEEE Transactions on Fuzzy Systems, vol. 17, no. 2, pp. 279-290, 2009. 